\title{
Research on the Application of Building Information Model Based on BIM Technology
}

\author{
Aimin Wei \\ Guangzhou City Construction College, China \\ wenhua1711@qq.com
}

Keywords: BIM Technology, Building Information Model, Application

\begin{abstract}
BIM is a new term in the construction industry in recent years and it is also the inevitable outcome of the development of information technology in the construction industry. The full application of BIM technology will generate immeasurable impact of scientific and technological progress of construction, greatly improving the degree of integration of construction projects and eventually has a huge benefit for the development of the construction industry. In this paper, we discussed the difference between traditional CAD and BIM, the domestic software applications of BIM, several aspects of the value of BIM technology. And we proposed the future application prospects of BIM technology.
\end{abstract}

\section{Introduction}

BIM (Building Information Modeling) is based on the relevant information and data as a basis for building projects model, were established architectural models, real information through digital simulation of building information possessed. It has visualization, coordination, simulation, optimize and can plot of five characteristics. In recent years, there is the inevitable product of a new term in the construction industry, as well as the development of information technology in the construction industry. Full application of BIM technology, will generate construction immeasurable impact of scientific and technological progress, greatly improving the degree of integration of construction projects, a huge benefit for the development of the construction industry. With the widely application of BIM technology in the country, China's construction industry is bound to have a transformative development and leap, it will bring many changes and optimization ideas, work, work processes, etc. for the construction industry practitioners. American National Standard for BIM meaning made the following four levels of interpretation: a one facility (building projects) digital representation of physical and functional characteristics; b. A shared knowledge resource; c a share on this... information infrastructure, provide a reliable basis for all decision-making facilities from the beginning of the whole concept of life-cycle process; at different stages of the project by the different stakeholders in the BIM insert, extract, update and modify the information to support and reflect its respective responsibilities of collaboration.[1]

\section{The Research on BIM in Domestic}

In recent years, China's construction industry, academics and research institutions related to BIM technology application in our country pay a sweat, mainly the implementation of research in BIM, China BIM Standards and technology aspects. BIM implementation research includes the implementation strategy of implementing Model, Study ways to promote the implementation of the framework and BIM technology. Our special issue BIM Standards from universities to government level BIM standards organizations subject cohort study. BIM technology application in China first place in the design stage, the three-dimensional visualization of the design results show much the owner, the contractor's favor. 


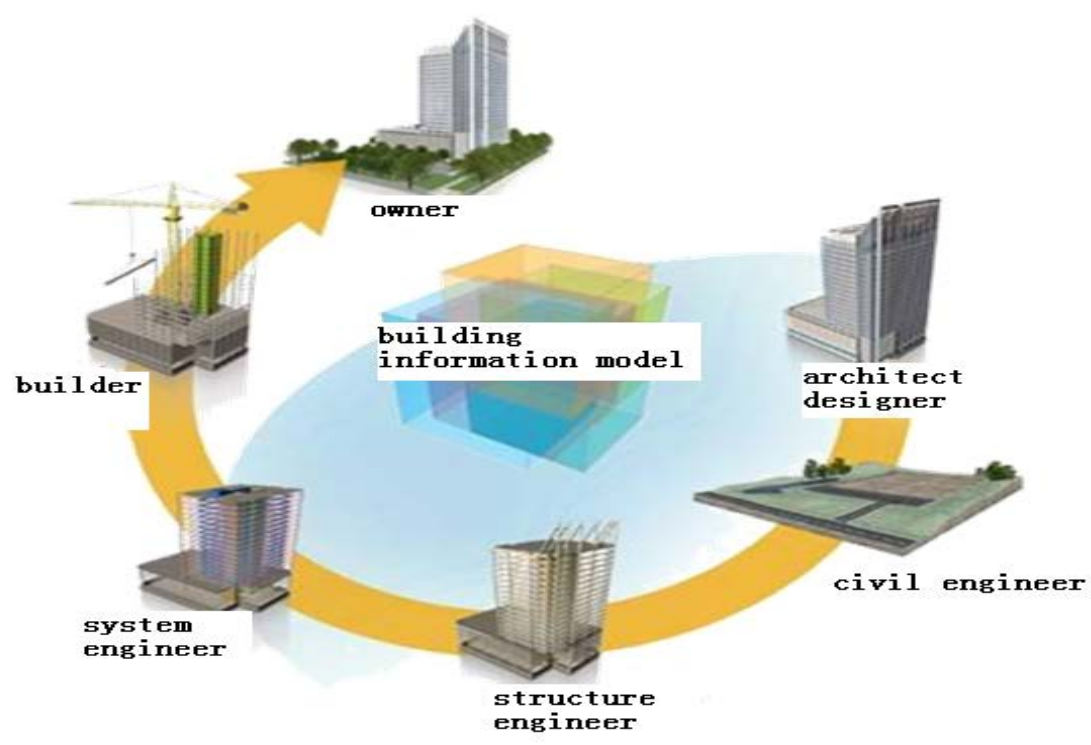

Fig. 1 The BIM in the whole life cycle of buildings

\section{The Difference between Traditional CAD and BIM}

The traditional AutoCAD software is generally based on two-dimensional graphic design. Build three-dimensional models (AutoCAD 3D modeling software comes with many defects) by Sketchup modeling software, such as when doing renderings, but the two are independent. In the BIM systems can be Sketchup, Rhino and FromZ information input BIM modeling software into the core, the core can also be used directly BIM modeling software to model the (complex massing studies and experience of the early stages of building design when modeling, using Sketchup software is more convenient). This will not affect the independent software flexibility while using more conducive to the consistency and integrity of project information. Three-dimensional model for building energy analysis design automation, intelligence provides a platform. BIM's 3D parametric design with specialized three-dimensional design tools, real-time visualization, a more advanced model of collaborative design and create the schedule automatic underlay construction detail by the model, supporting the analysis and simulation design tools. BIM technology to improve the architectural design phase information and three-dimensional space also allows designers richer design inspiration. BIM to improve the system and shorten the design period, designers do not have to blindly bury more time for drawing and design innovation. Traditional two-dimensional CAD design construction plans cannot express some complex modeling and BIM through 3D parametric design can perfectly express the designer's creativity [2].

Before contacting BIM, the entire construction industry already have experience in the successful application of CAD, so copy then popularized CAD experience to implement today's BIM application is the most natural thing, this experience is very simple:

The first step: send a few employees to accept the CAD software operating outside the training (or ask the teacher training to the corporate staff training);

Step two: the first to get rid of employees to participate in learning drawing board, with a CAD drawing;

The third step: step by step training and other staff to lead the company to get rid of the drawing board.

Features CAD applications is only one person, even if an enterprise will use a CAD software, other people will not use, efficiency and quality of the person (or at least drawing quality) compared with their original high compared to some time with the drawing board under normal circumstances is not bad as long as the professional competence, then naturally quite high professional competence than other employees, the results of this phenomenon, of course, other employees also 
requires companies to give their computer equipped with CAD drawings, line engineering and technical personnel of their own to use CAD initiative does not require much guidance and encouragement, then the greatest difficulty for businesses is much hardware and software investments.

We currently have universal BIM and CAD technology used for comparison, we find that CAD is basically a software thing, and BIM is not just a software thing; CAD basically just changed a tool, rather than just BIM for a tool to do; CAD users showed more personal matter, and BIM is not just one thing; CAD is basically just for a medium, and BIM is not just a medium change things.

That is, today, when companies began BIM applications, the principal contradiction and the main aspects related to the main contradiction of the year, when the popularity of CAD has changed a lot. First, from the technical point of view to analyze, CAD can independently achieve its core values (rejection drawing board, and thus obtained to improve the efficiency and quality of cartographic) employed by each individual, and each individual practitioners BIM by individuals unable to meet its basic core Values (improve the quality and efficiency of the overall project); followed up from the economic analysis, software and hardware prices have now generally and the industry average monthly salary of an order of magnitude, design firms average annual output of about 30 million, the implementation of BIM major cost by popularity during the year CAD hardware costs become today's human resource costs, and opportunity costs arising there from (some people some time to complete the project cannot proceed normally)[3]

BIM successful implementation of enterprise applications cannot rely on their own staff alone separately to realize the need for relevant professional organizations and a number of teams together to explore; BIM application for time to improve project quality and efficiency purposes is also needed to replace than the CAD map time required for more long plate, investment in time means that benefits can be obtained before the application of BIM longer needed. These characteristics determine the enterprises should be converted into their BIM effective productivity must be relatively systematic and complete plans and procedures otherwise would not be much chance of success, or the time required will be longer, the cost will be higher, the risk will be greater.

\section{The Domestic Software Applications of BIM}

In 2013, organized by the China Construction Industry Association, China Association for Engineering Construction Quality Management Branch hosted the first "Project BIM Application Contest", the results of 570 entries were received and these results reflect the current construction of basic business applications BIM Technology made new progress. China Construction Industry Association Construction Quality Management Branch of the spirit of justice, fairness and openness, re-evaluation of the initial evaluation by experts after the final assessment, the final selection of the first construction BIM application contest prize 6, 20 second prize, third Award 34 Award of Excellence 69.

(1)The results of the use of 26 award-winning software to covering the basic software used domestic construction, design, owners of companies are currently using.

(2)There are six outcomes (accounting for $6 / 26=23 \%$ ) and domestic use of domestic secondary development of software, including the self-developed software (refer to no marketing of proprietary software, in fact number three, here comes down to one class), Glodon, VR systems, Brownsville, Luban, Hongye BIM. Foreign software in terms of both the role of the number is predominant.

(3)There are 17 achievements $(17 / 26=65 \%)$ using Autodesk's Revit and Navisworks, 9 using tekla (accounting for 9/26 = 35\%), 7 using Rhino (accounting for 7/26 = 27\%), 4 using MagiCAD (accounting for $4 / 26=15 \%$ ). From the point of view that the popularity of Revit and Navisworks highest special steel and electromechanical applications Tekla and MagiCAD advantage. The results of the use of Rhino's award-winning high proportion of the ratio of the award-winning achievements illustrate the complex shape of the project.

(4)PKPM is the most widely popular structural design software, but only a small amount of the award-winning achievements fill out the use of PKPM, explain most of the participating teams have 
not put PKPM treated as BIM software. The similar situation are also AutoCAD and 3DS MAX.

(5)Bentley absence of a product, prize description and results of the infrastructure in the field of plant design to participate in this competition the number is still relatively small, especially in the construction industry, plant design is the use of information technology model (used to refer to 3D plant design) first field, there should be a lot of lessons worth learning in other areas of the universal application of BIM process [4]

\section{The Value of BIM Technology}

The construction sector to solve the current bottleneck of information technology to create a single source of engineering data

Each project participants use a single source of information to ensure the accuracy and consistency of information to realize the project participating information exchange and sharing between the parties. Fundamentally solve the project carried out between the parties involved, "information gap" and application systems for information exchange to form the "islands of information" issue paper-based methods. Promote the application of modern CAD technology. Full support for digitization, using different methods of engineering design, as far as possible the use of automated design technology, design integration, networking and intelligent. Promote the building life cycle management, construction engineering properties of the various stages of life, integrate management of safety, schedule and cost, of the total cost of the construction project life cycle, energy consumption, environmental impact analysis, forecasting and control.

Engineering BIM-based implementation of the three-dimensional design 3D models can be automatically generated based on a variety of graphics and documents, and is always associated with the model logic, when the model changes, graphics and documents associated with it will automatically update; the object created in the design process there is a built-in logic associated relationship, when an object changes, associated with the object changes. Professional CAD system can obtain the required design parameters and related information from the information model, no need to repeat data entry, avoid data redundancy, ambiguity and error. A professionally designed object is modified and the other specializing in the design of the object will be updated. Collision detection to achieve the design, energy analysis, cost forecasting [5].

\section{Conclusions}

We are in a period full of technological changing. The BIM brings not only the impact of technology, but also the impact on industry tradition. How to deal with this change correctly will be related to the future development and China's international status of design industry and the CAD industry. We have to combine Chinese characteristics seriously, combined with practical, work hard in practice, have the courage to explore to embark on a new development path as soon as possible.

\section{References}

[1] Information on http://baike.baidu.com/view/1281360.htm?fr=aladdin

[2] G.P. He: Information Technology in Civil Engineering and Construction, Vol. 2 (2011) No 3, p.114-118

[3] X.J. Xue: Structural Engineer, Vol. 1(2011) No 27, p. 14-18

[4] J. Qin: Architecture Technique, Vol. 3(2011). No10, p.160-163

[5] Information on http://blog.sina.com.cn/s/blog_620be62e0101dpdk.html 